\title{
Kinematic design of a finger abduction mechanism for an anthropomorphic robotic hand
}

\author{
L.-A. A. Demers and C. Gosselin \\ Département de génie mécanique, Université Laval, Québec, QC, G1V 0A6, Canada
}

Received: 25 February 2010 - Revised: 18 June 2010 - Accepted: 19 August 2010 - Published: 8 February 2011

\begin{abstract}
This paper presents the kinematic design of an abduction mechanism for the fingers of an underactuated anthropomorphic robotic hand. This mechanism will enhance the range of feasible grasps of the underactuated hand without significantly increasing its complexity. The analysis of the link between the index finger and the third finger is first assessed, where the parameters are studied in order to follow the amplitude constraint and to minimize the coordination error. Then, the study of the mechanism joining the third finger and the little finger is summarized. Finally, a prototype of the finger's abduction system is presented.
\end{abstract}

This paper was presented at the IFToMM/ASME International Workshop on

Underactuated Grasping (UG2010), 19 August 2010, Montréal, Canada.

\section{Introduction}

This paper presents work currently underway aiming at the design of an underactuated hand for an anthropomorphic robot. Underactuation has been chosen to simplify the control of this hand and to minimize its cost in order to facilitate its use by a humanoid robot. The 16-degree-of-freedom hand will therefore need four actuators to control its closing and opening motion as well as its abduction movement. The analyses of underactuation in the fingers and thumb were already presented (Demers and Gosselin, 2009, 2010). In order to maximize the range of feasible grasps, a mechanism for the abduction of the fingers is developed here. Abduction is the action of pulling a member away from a median axis. According to Boutan and Casoli (2005), a typical grasp, the spherical grip, is associated with maximum separation of fingers "made in relation to the anatomical axis of the hand through the 3rd ray", i.e., the middle finger. Therefore, the abduction mechanism of the fingers should leave the middle finger fixed. Thereby, the thumb could perform a pinch with it when they are in opposition.

Correspondence to: C. Gosselin (gosselin@gmc.ulaval.ca)

\section{Prescribed abduction movements}

In regard to measuring the magnitude of the spacing between the fingers, no source using a universal goniometer was found by Norkin and White (2009). For their part, Smahel and Klímová (2004) traced the contour of the fingers apart to measure the maximum angle between adjacent fingers. Also, Gurbuz et al. used an electronic inclinometer to measure active abduction of metacarpophalangeal joints (Gurbuz et al., 2006). Since no trend is apparent from these articles, we used the method proposed by Smahel and compared our results with those of other studies. We then concluded that a maximal abduction of $25^{\circ}$ between each finger would be sufficient, which represents a range of $50^{\circ}$ for the little finger.

It was also noted in the literature (Tubiana and Thomine, 1990; Kapandji, 2005) that the rays, the extensions of the fingers, all converge to a point at the base of the palm when the fingers are fully abducted, as illustrated in Fig. 1. Added to the fact that their width limits the minimum distance between them, it is possible to calculate the position of the axes of rotation, $O_{0, i}$, for the abduction of the metacarpals. Assuming that the distance between the centres of the fingers must be $25.4 \mathrm{~mm}$ and that the maximum abduction be $25^{\circ}$, we can calculate the distance from the meeting point $p_{0}$ of the rays on the middle finger's axis, that is $54.47 \mathrm{~mm}$. These dimensions are shown in Fig. 1. Furthermore, the metacarpophalangeal joints, $O_{1, i}$, in outspread position must be equidistant from the point of intersection of rays, $p_{0}$. For fingers of same 


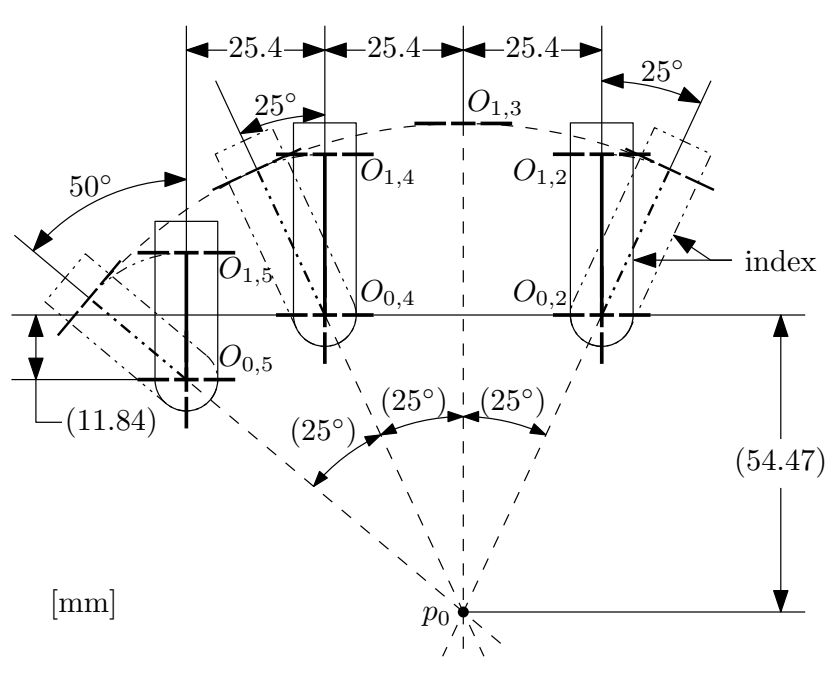

Figure 1. Abduction movement of the metacarpals.

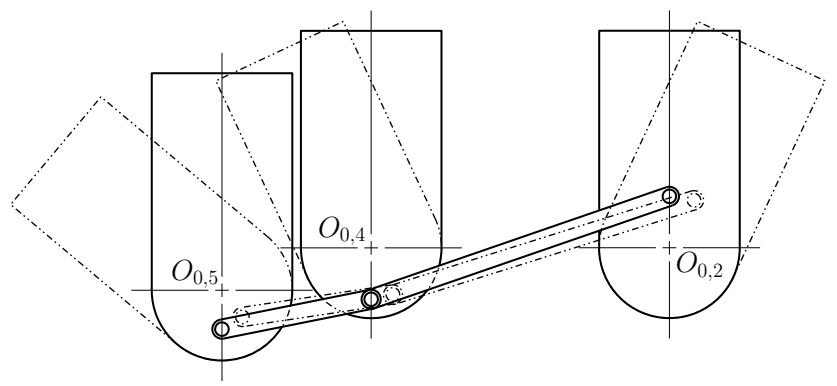

Figure 2. Linkage connecting the metacarpals.

dimension this specification allows the tips to be at the same distance during the closing, which is useful for a spherical grip. This feature is not necessary when making a cylindrical grip where the fingers are adjacent and wrapped around the object.

In order to simplify the architecture of the hand, abduction of the fingers and thumb should be performed with a single actuator. The mechanism of abduction of the thumb was presented in another article (Demers and Gosselin, 2010), but it is important to emphasize that its metacarpal is driven by a worm gear. This input can also be used to drive the abduction of the fingers. The design of the mechanism for the abduction of the fingers therefore consists in developing a link between the index and ring fingers, followed by a link between the ring and little fingers. Since simplicity and stiffness in the connection is required for a stable spherical grasp, two 4-bar mechanisms will be used, as shown in Fig. 2.

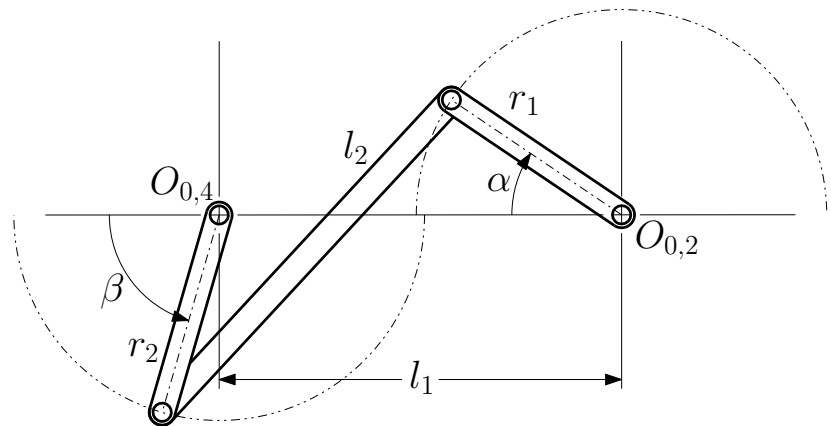

Figure 3. Linkage connecting the metacarpals of the index and ring fingers.

\section{Linkage between the index and ring fingers}

Since a 4-bar mechanism has been chosen to connect the index and ring fingers, it is now interesting to analyze the relationship between the input and output angles, that is to say between $\alpha$ and $\beta$ according to Fig. 3. We must thus determine the parameters of the mechanism that will allow the ring finger to follow the abduction of the index, in other words the range of motion $\Delta \beta$ between the initial and final position of the ring finger is equal to the range of motion of the index, $\Delta \alpha$. Also, it is preferable that this abduction be performed with a somewhat synchronized motion of the fingers, so $\delta \beta \approx \delta \alpha$ during the trajectory.

With Fig. 3, it is possible to establish that

$l_{2}^{2}=\left(r_{2} \cos \beta+l_{1}-r_{1} \cos \alpha\right)^{2}+\left(r_{1} \sin \alpha+r_{2} \sin \beta\right)^{2}$

and using equal radii, $r_{1}=r_{2}=r$, one can obtain

$A_{\beta} \cos \beta+B_{\beta} \sin \beta=C_{\beta}$

where

$A_{\beta}=\cos \alpha-\frac{l_{1}}{r}$

$B_{\beta}=-\sin \alpha$

$C_{\beta}=1+\frac{l_{1}^{2}-l_{2}^{2}}{2 r^{2}}-\frac{l_{1}}{r} \cos \alpha$.

In a typical linkage synthesis application, Eq. (1) is written for a series of prescribed input-output pairs until a sufficient number of conditions is obtained. The equations are then solved using, for instance, nonlinear least squares techniques. However, in this work, only a small set of constraints will first be imposed, thereby leaving some design freedom in the problem. Then, a detailed analysis will be performed in order to explore the possibility of minimizing the coordination error throughout the motion range, without arbitrarily prescribing a large number of input-ouput points.

Equation (2) is readily solved and leads to two values of angle $\beta$ associated with the input angle $\alpha$ for given link lengths. Since the abduction range of motion of the index and 
ring fingers should both be equal to $25^{\circ}$, thus $\Delta \beta=\Delta \alpha=25^{\circ}$. It is thus necessary to establish a relationship between these amplitudes and ratios $l_{1} / r$ or $l_{2} / r$ in order to synthesize a mechanism that will meet this condition.

\subsection{Amplitudes of abduction}

Using the initial, $\beta_{0}$, medial, $\beta_{1}$, and final, $\beta_{2}$, angles of the ring finger as well as those of the index, $\alpha_{0}, \alpha_{1}$ and $\alpha_{2}$, relationships between these angles can be established.

$\beta_{2}-\beta_{0}=\Delta \beta=\Delta \alpha=\alpha_{2}-\alpha_{0}$

Consequently

$\beta_{2}=\beta_{1}+\frac{\Delta \beta}{2}=\beta_{1}+\frac{\Delta \alpha}{2}$

$\beta_{0}=\beta_{1}-\frac{\Delta \beta}{2}=\beta_{1}-\frac{\Delta \alpha}{2}$

$\alpha_{2}=\alpha_{1}+\frac{\Delta \alpha}{2}$

$\alpha_{0}=\alpha_{1}-\frac{\Delta \alpha}{2}$.

It is important to note that the median angles $\alpha_{1}$ and $\beta_{1}$ do not necessarily occur simultaneously. Indeed, at this point, Eq. (2) is not required to be satisfied for $\alpha_{1}$ and $\beta_{1}$. Equation (2) can be rewritten to highlight the constant term for a given mechanism, which leads to

$\cos (\alpha+\beta)+\frac{l_{1}}{r}(\cos \alpha-\cos \beta)=1+\frac{l_{1}^{2}-l_{2}^{2}}{2 r^{2}}$.

From the initial configuration $\left(\alpha_{0}, \beta_{0}\right)$ to the final one $\left(\alpha_{2}, \beta_{2}\right)$, the left hand side of Eq. (11) must always give the same result for the mechanism to be assembled. Therefore, one can write

$$
\begin{aligned}
\cos \left(\alpha_{0}+\beta_{0}\right)+\frac{l_{1}}{r}\left(\cos \alpha_{0}-\cos \beta_{0}\right) \\
=\cos \left(\alpha_{2}+\beta_{2}\right)+\frac{l_{1}}{r}\left(\cos \alpha_{2}-\cos \beta_{2}\right) .
\end{aligned}
$$

Substituting Eq. (7) to Eq. (10) into Eq. (12), one can obtain

$0=-2 \sin \left(\alpha_{1}+\beta_{1}\right) \sin \Delta \alpha+2 \frac{l_{1}}{r}\left(\sin \beta_{1}-\sin \alpha_{1}\right) \sin \frac{\Delta \alpha}{2}$.

The latter equation is satisfied if either $\Delta \alpha=2 n \pi$ where $n \in \mathbb{N}$, so the mechanism does not move or makes $n$ turns, or $\sin \left(\alpha_{1}+\beta_{1}\right)=0$ and $\sin \beta_{1}-\sin \alpha_{1}=0$ for any $l_{1} / r$. The latter conditions are satisfied if and only if $\alpha_{1}+\beta_{1}=(2 n-1) \pi$. Since the four-bar mechanism is assembled using the inverted branch and $\Delta \beta=\Delta \alpha$, then it follows from Eq. (7) to Eq. (10) that

$\alpha_{1}+\beta_{1}=\pi$

$\alpha_{0}+\beta_{2}=\pi$

$\alpha_{2}+\beta_{0}=\pi$.

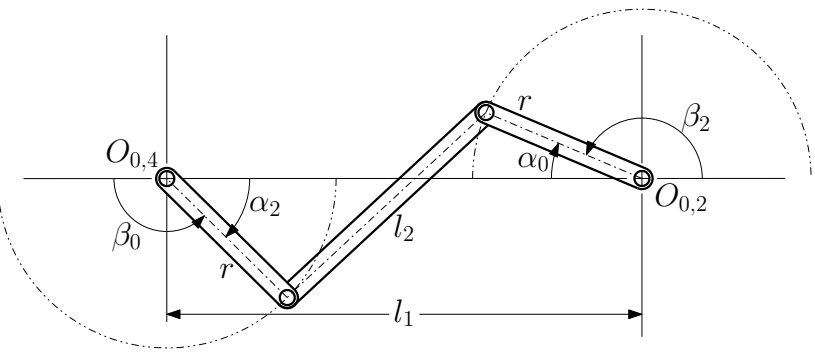

Figure 4. Linkage connecting the metacarpals of the index and ring fingers in its initial position $\left(\alpha_{0}, \beta_{0}\right)$.

Equations (15) and (16), described in Fig. 4, can then be substituted into Eq. (1), leading to

$\left(\frac{l_{2}}{r}\right)^{2}=\left(\frac{l_{1}}{r}-\cos \alpha_{0}-\cos \alpha_{2}\right)^{2}+\left(\sin \alpha_{0}+\sin \alpha_{2}\right)^{2}$

which can be rewritten as

$\left(\frac{l_{2}}{r}\right)^{2}=\left(\frac{l_{1}}{r}\right)^{2}-4 \frac{l_{1}}{r} \cos \alpha_{1} \cos \frac{\Delta \alpha}{2}+4 \cos ^{2} \frac{\Delta \alpha}{2}$.

For a given range of abduction $\Delta \alpha$, it is then possible to use the above equation to calculate the ratio $l_{2} / r$ with $l_{1} / r$ and $\alpha_{1}$.

\subsection{Minimizing the coordination error}

In addition to imposing that the difference between the initial and final position of the ring finger is equal to that of the index, i.e. $\Delta \beta=\Delta \alpha$, it is required that the abduction motion of the fingers be somewhat synchronized, that is to say $\delta \beta \approx \delta \alpha$ over the motion range. It is therefore necessary to minimize the coordination error $e_{\beta}$ between the orientation of the ring and index fingers. This can be described as the absolute difference between the angular displacements, i.e.

$e_{\beta}=\left|\left(\beta-\beta_{0}\right)-\left(\alpha-\alpha_{0}\right)\right|=\left|\beta-\alpha-\left(\beta_{1}-\alpha_{1}\right)\right|$

and with Eq. (14):

$e_{\beta}=\left|\beta-\alpha-\pi+2 \alpha_{1}\right|$.

The error varies with the configuration of the mechanism, i.e. according to $\alpha$ and $\beta$. The parameters that can be modified in order to minimize the error $e_{\beta}$ are $l_{1} / r$ and $\alpha_{1}$, since $\Delta \alpha$ is constrained and $\alpha$ varies according to $\alpha_{1}-\Delta \alpha / 2 \leq \alpha \leq \alpha_{1}+\Delta \alpha / 2$. The minimization of the error can be performed in several ways.

It is first interesting to trace the contours of the coordination error $e_{\beta}$ for $\alpha_{0} \leq \alpha \leq \alpha_{2}$ and various $\alpha_{1}$ using a prescribed ratio $l_{1} / r$, as shown in Fig. 5. To minimize the error on the interval $\alpha_{0} \leq \alpha \leq \alpha_{2}$, we can examine the evolution of the mean and the maximum error on this interval. Figure 6 is obtained using again $l_{1} / r=5$. It can be observed in Fig. 5 that a minimum can be achieved for the error $e_{\beta}$. The value of $\alpha_{1}$ for this minimum is denoted $\alpha_{g}$ in Fig. 6 and we note that 


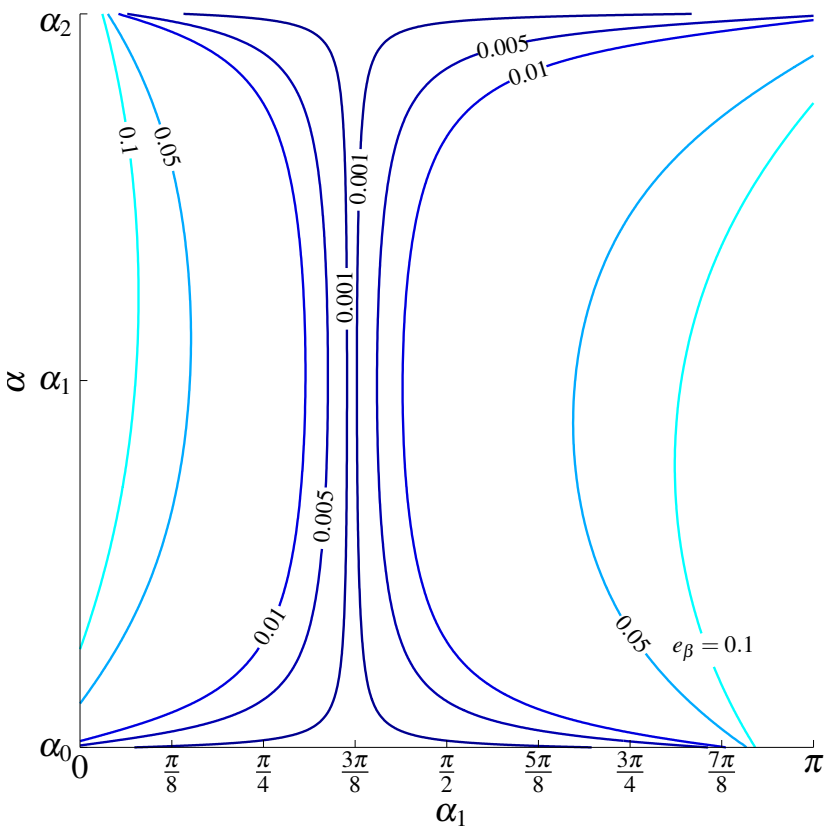

Figure 5. Coordination Error $e_{\beta}$ as a function of the median angle $\alpha_{1}$ for $\alpha_{0} \leq \alpha \leq \alpha_{2}$ if $l_{1} / r=5$.

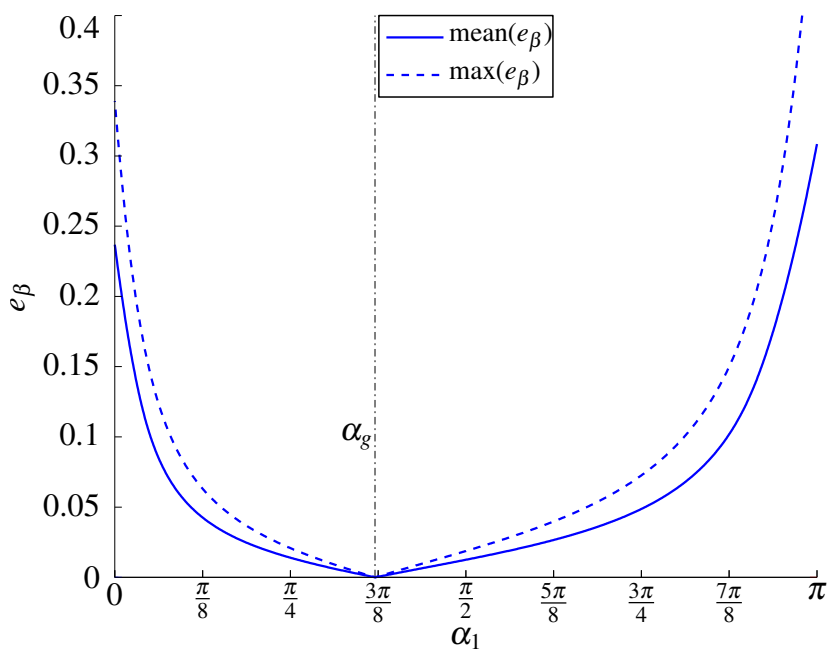

Figure 6. Mean and maximum error in the interval $\alpha_{0} \leq \alpha \leq \alpha_{2}$ as a function of $\alpha_{1}$ for $l_{1} / r=5$.

the maximum as well as the average error over the interval is then minimized.

Since the trend of coordination error can be followed with its maximum value as well as its mean, it is now possible to analyze the effects of changing $\alpha_{1}$ along with $l_{1} / r$, as shown in Fig. 7. Low levels contours $(0.01,0.025,0.04,0.055,0.07$, $0.085)$ were added to higher levels $(0.1,0.2,0.3,0.4,0.5)$ to indicate the rapid growth of the error following the deviation from the ideal value $\alpha_{g}$.

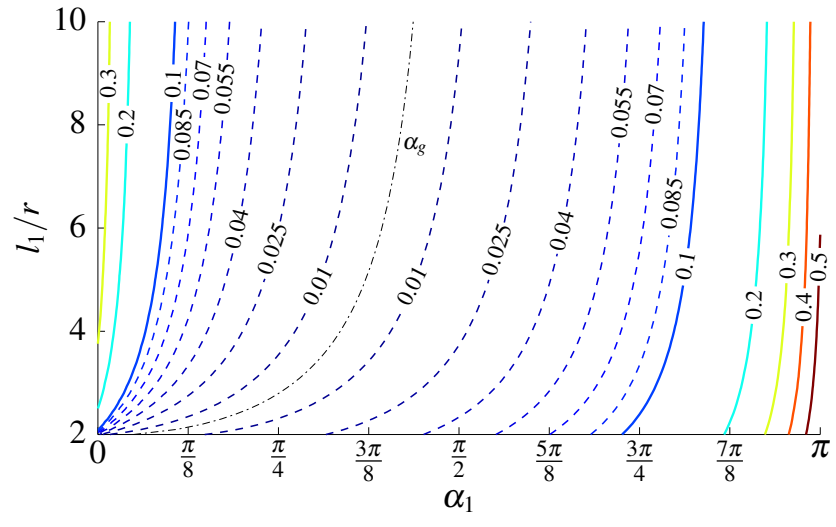

Figure 7. Maximum error in the interval $\alpha_{0} \leq \alpha \leq \alpha_{2}$ as a function of $\alpha_{1}$ and $l_{1} / r$.

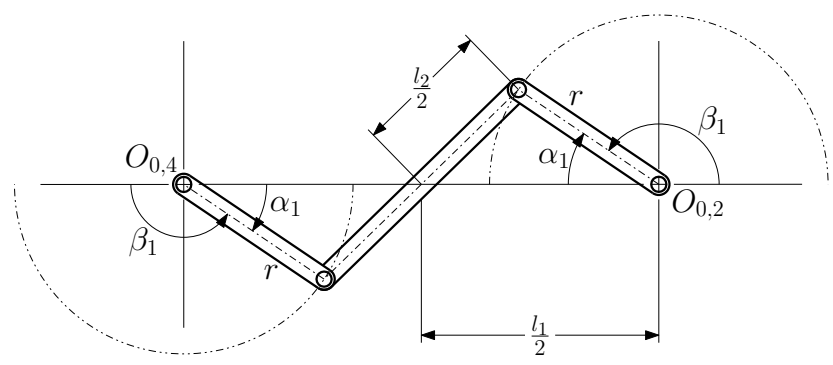

Figure 8. Linkage between the index finger and ring finger if the middle positions occur simultaneously.

Assuming that the median angles $\alpha_{1}$ and $\beta_{1}$ occur simultaneously and using Eq. (14), we can conclude that the centre of the link of length $l_{2}$ is located at the centre point between the two axes of rotation as sketched in Fig. 8. It is then possible to deduce the angle $\alpha_{1}$.

$\cos \alpha_{1}=\frac{1+\left(\frac{l_{1}}{2 r}\right)^{2}-\left(\frac{l_{2}}{2 r}\right)^{2}}{l_{1} / r}$

Using Eq. (21), one can factor Eq. (18) into two terms to emphasize its roots

$0=\left[\left(\frac{l_{1}}{r}\right)^{2}-\left(\frac{l_{2}}{r}\right)^{2}-4 \cos \frac{\Delta \alpha}{2}\right]\left[1-\cos \frac{\Delta \alpha}{2}\right]$

where we find again the trivial case $\Delta \alpha / 2=2 n \pi$. Besides, it is obvious that an equation to calculate $l_{2} / r$ can be obtained from the first term, which gives:

$\frac{l_{2}}{r}=\sqrt{\left(\frac{l_{1}}{r}\right)^{2}-4 \cos \frac{\Delta \alpha}{2}}$

and we can finally change Eq. (21) to calculate the angle $\alpha_{1}$ as a function of $\Delta \alpha / 2$ and $l_{1} / r$.

$\cos \alpha_{1}=\frac{1+\cos \frac{\Delta \alpha}{2}}{l_{1} / r}$ 


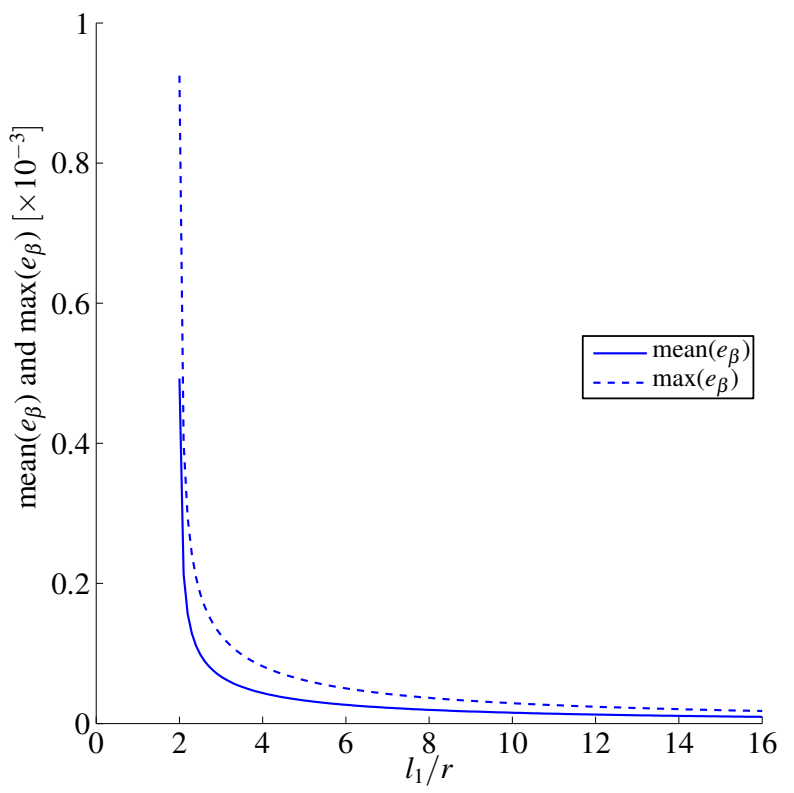

Figure 9. Mean and maximum error in the interval $\alpha_{0} \leq \alpha \leq \alpha_{2}$ according to $l_{1} / r$.

The median angle $\alpha_{1}$ obtained must be used if one wants to reduce the coordination error between the orientation of the ring finger and index finger. It is this angle, i.e. $\alpha_{g}$, which is indicated on Fig. 6 and Fig. 7. Indeed, if we use $\Delta \alpha=25^{\circ}$ and $l_{1} / r=5$, we obtain $\alpha_{1} \approx 1.164 \approx 3 \pi / 8$, which is the value of $\alpha_{g}$ already found for these parameters.

Since Eq. (24) gives a relationship between the two parameters of the optimization, minimizing the error $e_{\beta}$ on the interval $\alpha_{0} \leq \alpha \leq \alpha_{2}$ depends now only on the ratio $l_{1} / r$. It is then possible to obtain Fig. 9 by tracing the evolution of the mean and the maximum error following this parameter. It is obvious that for values of $l_{1} / r>3$ the maximum and average of the error $e_{\beta}$ become negligible $\left(\max \left(e_{\beta}\right)<0.13 \times 10^{-3}\right)$. Moreover, from $l_{1} / r=5$, the slope of the curve $\max \left(e_{\beta}\right)$ remains greater than $-0.015 \times 10^{-3}$. Since the distance $l_{1}$ between the axes of the index and ring fingers is equal to $50.8 \mathrm{~mm}$, we can assume that a ratio $l_{1} / r=5.08$ is feasible in practice, because the distance between two joints is then $r=10 \mathrm{~mm}$. For guidance, we obtain $l_{2} / r \approx 4.68$, thus $l_{2} \approx 46.8 \mathrm{~mm}$ and $\alpha_{1} \approx 1.171$.

The error obtained using this ratio is presented in Fig. 10 on a logarithmic scale. One can observe that the error is minimized over the interval $\alpha_{0} \leq \alpha \leq \alpha_{2}$. In fact, the magnitude of the error generated is negligible in practice. Moreover, since we have imposed $\Delta \beta=\Delta \alpha$, we obtain $e_{\beta}=0$ when $\alpha=\alpha_{0}$ and $\alpha=\alpha_{2}$. The error is also zero when $\alpha=\alpha_{1}$, because this angle is now calculated so that $\beta_{1}$ is achieved simultaneously with $\alpha_{1}$. With Eq. (19), it is obvious that the error is null if one uses $\alpha=\alpha_{1}$ and $\beta=\beta_{1}$. Finally, the evolution of the angle $\beta$ as a function of the angle $\alpha$ using $l_{1} / r=5$ is plotted in Fig. 11.

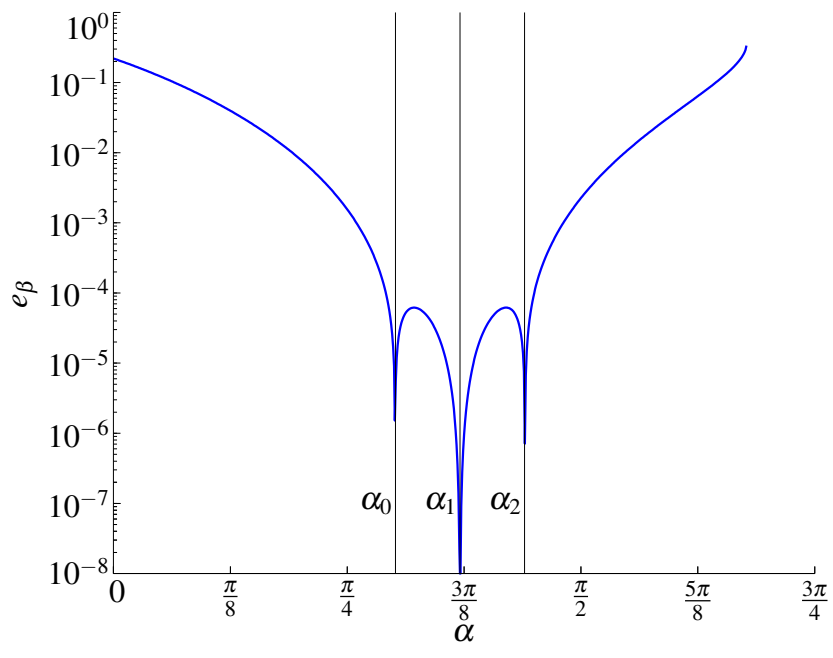

Figure 10. Error $e_{\beta}$ as a function of $\alpha$ with $l_{1} / r=5$.

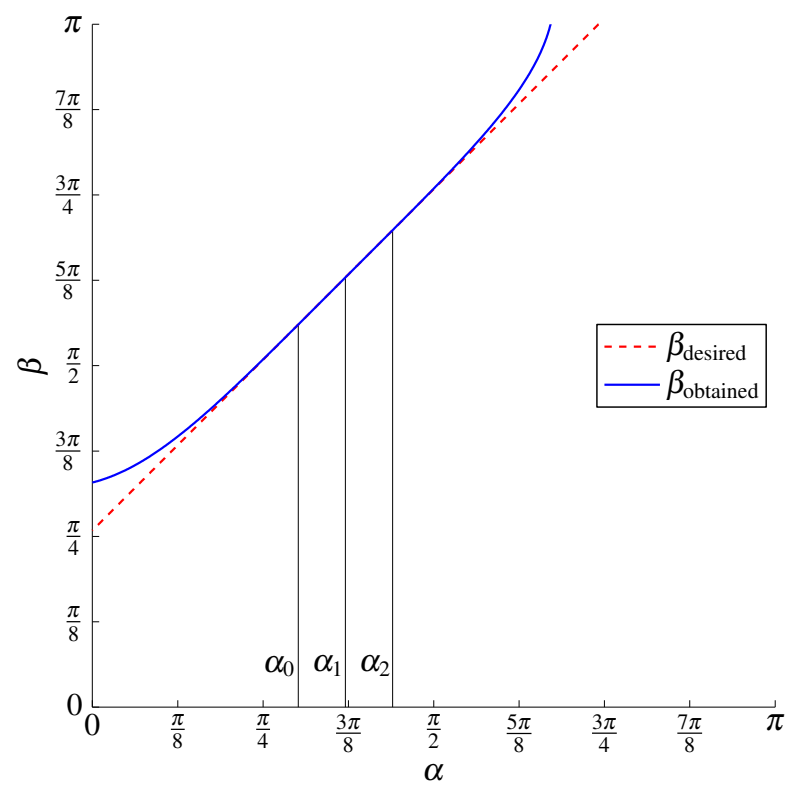

Figure 11. Angle $\beta$ desired and obtained as a function of angle $\alpha$ with $l_{1} / r=5$.

\section{Linkage between the ring finger and the little finger}

Since the parameters of the linkage between the index and ring fingers are fixed, it is now possible to analyze the linkage between the ring and little fingers, as shown in Fig. 12.

By following the same approach used to analyze the fourbar mechanism connecting the index and ring fingers, it is possible to determine from Fig. 12 that

$l_{4}^{2}=\left(r_{3} \cos \gamma+l_{3}-r_{2} \cos \beta\right)^{2}+\left(r_{3} \sin \gamma+l_{5}-r_{2} \sin \beta\right)^{2}$

which yields

$A_{\gamma} \cos \gamma+B_{\gamma} \sin \gamma=C_{\gamma}$ 


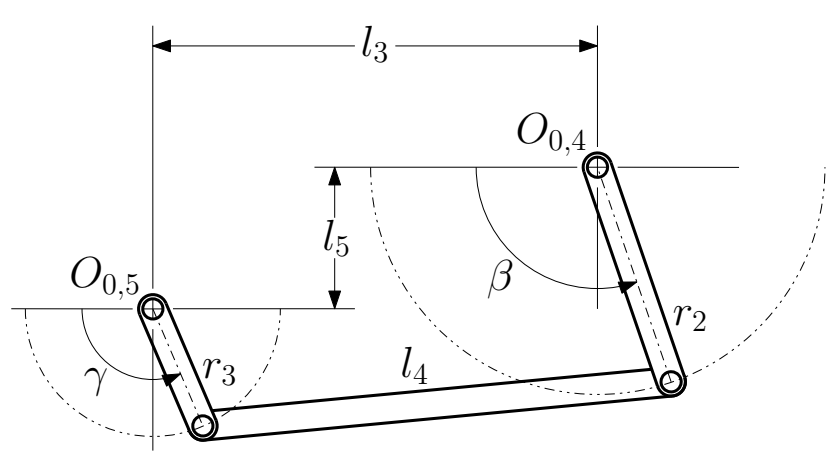

Figure 12. Linkage between the ring finger and the little finger metacarpals.

with

$A_{\gamma}=2 r_{3}\left(r_{2} \cos \beta-l_{3}\right)$

$B_{\gamma}=2 r_{3}\left(r_{2} \sin \beta-l_{5}\right)$

$C_{\gamma}=r_{2}^{2}+r_{3}^{2}+l_{3}^{2}+l_{5}^{2}-l_{4}^{2}-2 r_{2}\left(l_{3} \cos \beta+l_{5} \sin \beta\right)$.

Equation (26) is readily solved for $\gamma$ if all other parameters are given. Since $\beta_{1}$ and $r_{2}$ of this linkage are equal to $\beta_{1}$ and $r_{2}$ of the previous linkage in order to simplify the mechanism, it is preferable to use links of different lengths in order to keep parameter $r_{3}$ for the optimization.

Again, the median angle $\gamma_{1}$ giving the lowest error is first established, which will subsequently be used to determine $r_{3}$ and $l_{4}$. Since the amplitude of the range of motion for the little finger is $50^{\circ}$, we have $\Delta \gamma=2 \Delta \beta=50^{\circ}$.

\subsection{Amplitudes of abduction}

Initial, $\gamma_{0}$, medial, $\gamma_{1}$, and final, $\gamma_{2}$, angles of the little finger are assumed to be similar to those of the ring finger, $\beta_{0}, \beta_{1}$, $\beta_{2}$. This allows to establish relations similar to those derived in the previous section:

$\gamma_{2}-\gamma_{0}=\Delta \gamma=2 \Delta \beta=2\left(\beta_{2}-\beta_{0}\right)$

thus

$\gamma_{2}=\gamma_{1}+\frac{\Delta \gamma}{2}=\gamma_{1}+\Delta \gamma$

$\gamma_{0}=\gamma_{1}-\frac{\Delta \gamma}{2}=\gamma_{1}-\Delta \gamma$

$\beta_{2}=\beta_{1}+\frac{\Delta \beta}{2}$

$\beta_{0}=\beta_{1}-\frac{\Delta \beta}{2}$.

Again, the median angles $\beta_{1}$ and $\gamma_{1}$ do not necessarily occur simultaneously. Following the same method as in the previous case, it is possible to rewrite Eq. (26) as $g(\beta, \gamma)=l_{6}$ where

$$
\begin{aligned}
g(\beta, \gamma)= & r_{2} r_{3} \cos (\beta-\gamma)+l_{3}\left(r_{2} \cos \beta-r_{3} \cos \gamma\right) \\
& +l_{5}\left(r_{2} \sin \beta-r_{3} \sin \gamma\right) \\
l_{6}= & \frac{r_{2}^{2}+r_{3}^{2}+l_{3}^{2}+l_{5}^{2}-l_{4}^{2}}{2} .
\end{aligned}
$$

Since $l_{6}$ is constant for a given mechanism, the function $g(\beta, \gamma)$ must also be constant. Consequently, it is possible to use the two positions where the angles are known, i.e., the initial $\left(\beta_{0}, \gamma_{0}\right)$ and final $\left(\beta_{2}, \gamma_{2}\right)$ configurations, to find a relationship between $\gamma_{1}$ and $r_{3}$ not involving $l_{4}$.

One has:

$0=g\left(\beta_{0}, \gamma_{0}\right)-g\left(\beta_{2}, \gamma_{2}\right)$

which can be rewritten as

$$
\begin{aligned}
0= & r_{2}\left(r_{3} \sin \left(\gamma_{1}-\beta_{1}\right)+l_{3} \sin \beta_{1}-l_{5} \cos \beta_{1}\right) \sin (\Delta \beta / 2) \\
& +r_{3}\left(l_{5} \cos \gamma_{1}-l_{3} \sin \gamma_{1}\right) \sin \Delta \beta .
\end{aligned}
$$

If $\sin (\Delta \beta / 2) \neq 0$, i.e. $\Delta \beta \neq 2 n \pi$ where $n \in \mathbb{N}$, which implies that the mechanism does not move or makes $n$ complete turns, then it is possible to obtain

$$
\begin{aligned}
0= & r_{2}\left(r_{3} \sin \gamma_{1} \cos \beta_{1}-r_{3} \cos \gamma_{1} \sin \beta_{1}+l_{3} \sin \beta_{1}-l_{5} \cos \beta_{1}\right) \\
& +r_{3}\left(l_{5} \cos \gamma_{1}-l_{3} \sin \gamma_{1}\right) 2 \cos (\Delta \beta / 2)
\end{aligned}
$$

and finally

$\frac{r_{2}}{r_{3}}=\frac{r_{2} \sin \beta_{1}-2 l_{5} \cos \frac{\Delta \beta}{2}}{l_{3} \sin \beta_{1}-l_{5} \cos \beta_{1}} \cos \gamma_{1}+\frac{-r_{2} \cos \beta_{1}+2 l_{3} \cos \frac{\Delta \beta}{2}}{l_{3} \sin \beta_{1}-l_{5} \cos \beta_{1}} \sin \gamma_{1}$.

Knowing the values of $\Delta \beta, \beta_{1}, r_{2}, l_{3}$ and $l_{5}$, it is possible to use Eq. (40) to calculate $r_{3}$ depending of $\gamma_{1}$ and vice versa.

With $r_{3}$ and $\gamma_{1}$, it is then possible to calculate $l_{4}$ using the loop closure equation of the mechanism in the initial position $\left(\beta_{0}, \gamma_{0}\right)$ :

$$
\begin{aligned}
l_{4}^{2}= & \left(r_{3} \cos \left(\gamma_{1}-\Delta \beta\right)+l_{3}-r_{2} \cos \left(\beta_{1}-\Delta \beta / 2\right)\right)^{2} \\
& +\left(r_{3} \sin \left(\gamma_{1}-\Delta \beta\right)+l_{5}-r_{2} \sin \left(\beta_{1}-\Delta \beta / 2\right)\right)^{2} .
\end{aligned}
$$

This allows to plot the lengths $r_{3}$ and $l_{4}$ in terms of $\gamma_{1}$ as presented in Fig. 13, where $\Delta \beta=25^{\circ}, \beta_{1}=1.97, r_{2}=10 \mathrm{~mm}$, $l_{3}=25.4 \mathrm{~mm}$ and $l_{5}=11.8 \mathrm{~mm}$ were used.

Knowing for $\gamma_{1}$ the value of $r_{3}$ and $l_{4}$, it is possible to calculate angle $\gamma$ using Eq. (26) as well as the coordination error $e_{\gamma}$ between the little finger and the ring finger, where

$e_{\gamma}=\left|\left(\gamma-\gamma_{0}\right)-2\left(\beta-\beta_{0}\right)\right|=\left|\gamma-2 \beta-\left(\gamma_{1}-2 \beta_{1}\right)\right|$.

It is finally possible to see the coordination error on the interval $\beta_{0} \leq \beta \leq \beta_{2}$ according to the median angle $\gamma_{1}$ chosen, as shown in Fig. 14. Again, it is interesting to see the evolution of the mean and the maximum error on the useful range of $\beta$ : this can be done with Fig. 15. The error is then minimized when $\gamma_{1}=\gamma_{1 g}$, which represents the median angle for which the error becomes zero when $\beta=\beta_{1}$, as seen in Fig. 14 . 

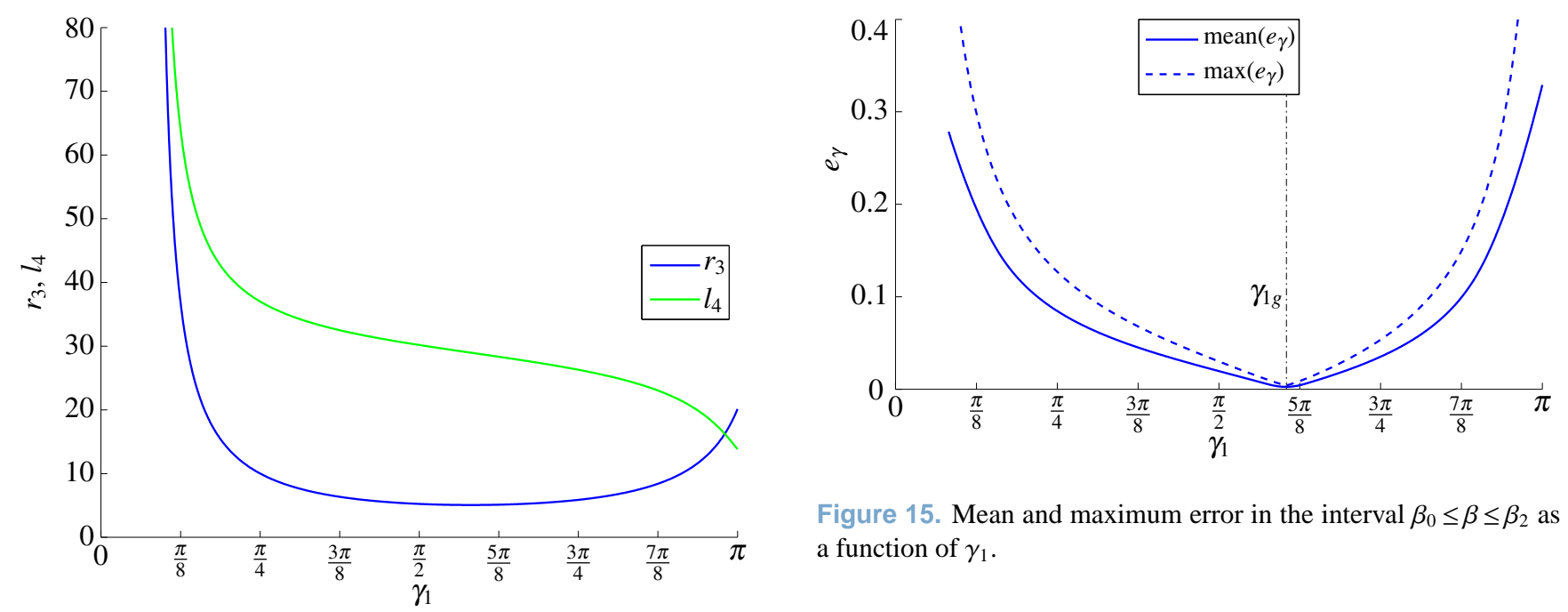

Figure 15. Mean and maximum error in the interval $\beta_{0} \leq \beta \leq \beta_{2}$ as a function of $\gamma_{1}$.

Figure 13. Lengths $r_{3}$ and $l_{4}$ as a function of angle $\gamma_{1}$.
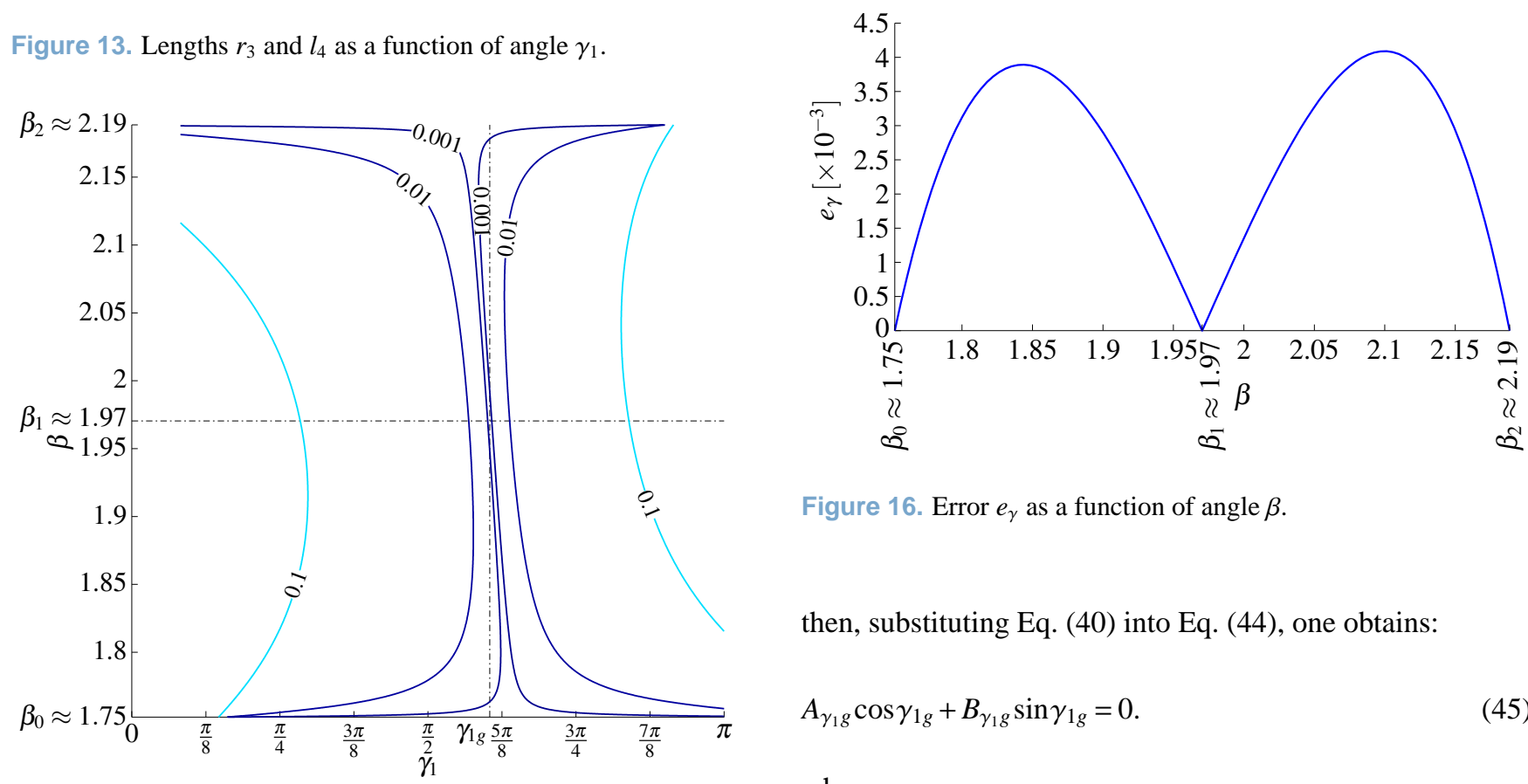

Figure 16. Error $e_{\gamma}$ as a function of angle $\beta$.

then, substituting Eq. (40) into Eq. (44), one obtains:

$A_{\gamma_{1} g} \cos \gamma_{1 g}+B_{\gamma_{1} g} \sin \gamma_{1 g}=0$

Figure 14. Error $e_{\gamma}$ as a function of $\gamma_{1}$ and $\beta$.

One can obtain $\gamma_{1 g}$ with $0=g\left(\beta_{1}, \gamma_{1 g}\right)-g\left(\beta_{0}, \gamma_{0}\right)$, i.e.,

$$
\begin{aligned}
0= & r_{2} r_{3} \cos \left(\beta_{1}-\gamma_{1 g}\right)+l_{3}\left(r_{2} \cos \beta_{1}-r_{3} \cos \gamma_{1 g}\right) \\
& +l_{5}\left(r_{2} \sin \beta_{1}-r_{3} \sin \gamma_{1 g}\right)-r_{2} r_{3} \cos \left(\beta_{0}-\gamma_{0}\right) \\
& -l_{3}\left(r_{2} \cos \beta_{0}-r_{3} \cos \gamma_{0}\right)-l_{5}\left(r_{2} \sin \beta_{0}-r_{3} \sin \gamma_{0}\right)
\end{aligned}
$$

which can be rearranged as

$$
\begin{aligned}
0= & \cos \gamma_{1 g}\left(r_{2}\left(\cos \beta_{1}-\cos \beta_{2}\right)-l_{3}(1-\cos \Delta \beta)-l_{5} \sin \Delta \beta\right) \\
& +\sin \gamma_{1 g}\left(r_{2}\left(\sin \beta_{1}-\sin \beta_{2}\right)+l_{3} \sin \Delta \beta-l_{5}(1-\cos \Delta \beta)\right) \\
& +\frac{r_{2}}{r_{3}}\left(l_{3} \cos \beta_{1}-l_{3} \cos \beta_{0}+l_{5} \sin \beta_{1}-l_{5} \sin \beta_{0}\right)
\end{aligned}
$$

where

$$
\begin{aligned}
A_{\gamma_{1} g}= & r_{2}\left(\cos \beta_{1}-\cos \beta_{2}\right)-l_{3}(1-\cos \Delta \beta)-l_{5} \sin \Delta \beta \\
& +d_{\gamma_{1} g}\left(r_{2} \sin \beta_{1}-2 l_{5} \cos \Delta \beta / 2\right) \\
B_{\gamma_{1} g}= & r_{2}\left(\sin \beta_{1}-\sin \beta_{2}\right)+l_{3} \sin \Delta \beta-l_{5}(1-\cos \Delta \beta) \\
& +d_{\gamma_{1} g}\left(-r_{2} \cos \beta_{1}+2 l_{3} \cos \Delta \beta / 2\right) \\
d_{\gamma_{1} g}= & \frac{l_{3}\left(\cos \beta_{1}-\cos \beta_{0}\right)+l_{5}\left(\sin \beta_{1}-\sin \beta_{0}\right)}{l_{3} \sin \beta_{1}-l_{5} \cos \beta_{1}} .
\end{aligned}
$$

which is readily solved for $\gamma_{1 g}$. Using the same parameters as used for Fig. 13, we obtain $\gamma_{1 g} \approx 1.90$ and the associated values $r_{3} \approx 5.1 \mathrm{~mm}$ and $l_{4} \approx 28.6 \mathrm{~mm}$. The error $e_{\gamma}$ is presented in Fig. 16. One can observe that the error is zero at $\beta=\beta_{0}, \beta_{1}$ and $\beta_{2}$. Finally, the angle $\gamma$ is plotted in Fig. 17 as a function of angle $\beta$. 


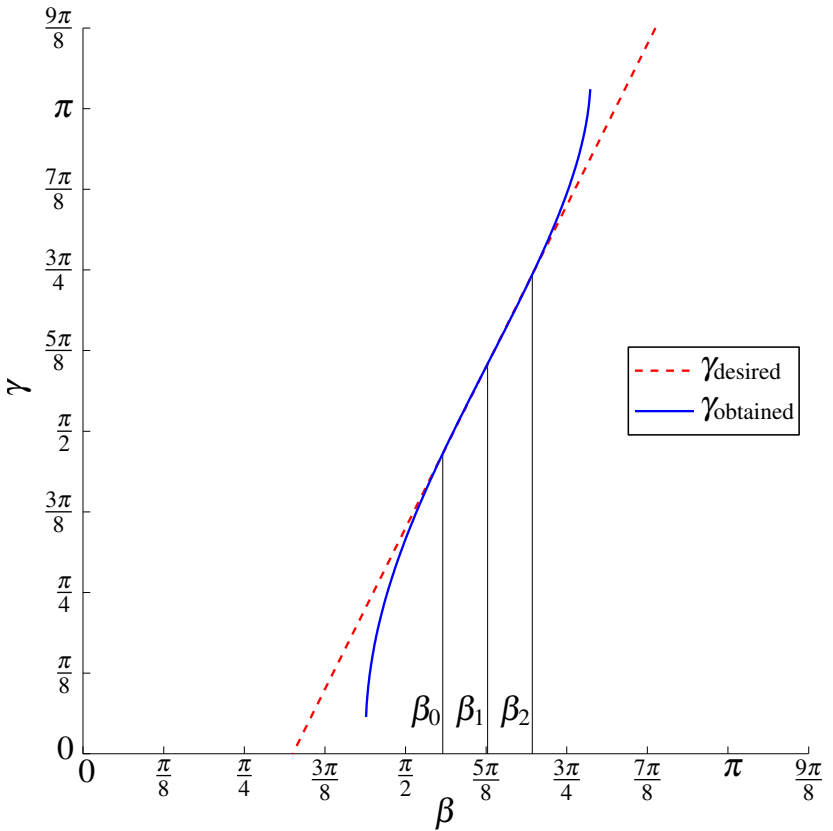

Figure 17. Angle $\gamma$ desired and obtained according to $\beta$.

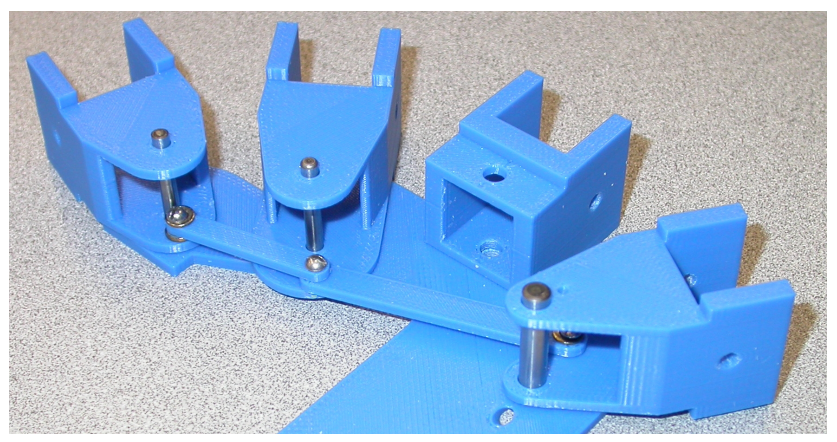

Figure 18. Prototype of the finger abduction system.

\section{Prototype}

Using the values found in this study, the prototype shown in Fig. 18 was built with rapid prototyping. One can note the similarity with the schematic presented in Fig. 2, on which the fixed middle finger metacarpal has been added. Again, the input consisting of a worm gear connecting with the index metacarpal is not shown. This mechanism will enhance the range of feasible grasps of the underactuated hand without significantly increasing its complexity.

\section{Conclusions}

The kinematic design of the finger abduction mechanism for an underactuated anthropomorphic robotic hand has been presented. The parameters of the linkage between the index finger and the third finger has been analyzed, followed by those of the linkage between the ring finger and the little finger. We therefore obtain a simple architecture that minimizes the coordination error among the abduction angles.

A robotic hand using this abduction mechanism is being built. It will use underactuation within its fingers and among them and will be tendon-driven (Demers and Gosselin, 2009, 2010). Testing of its grasping capabilities will then be conducted in order to validate this design.

Acknowledgements. This work was supported by the Fonds québécois de la recherche sur la nature et les technologies (FQRNT), the Natural Sciences and Engineering Research Council of Canada (NSERC) as well as by the Canada Research Chair Program.

Edited by: J. L. Herder

Reviewed by: two anonymous referees

\section{References}

Boutan, M. and Casoli, V.: Mains \& Préhensions entre fonctions et anatomie, Sauramps médical, Montpellier, 2005.

Demers, L.-A. A. and Gosselin, C.: Kinematic design of an ejection-free underactuated anthropomorphic finger, in: Proceedings of the IEEE International Conference on Robotics and Automation (ICRA 2009), Kobe, Japan, 12-17 May 2009, 20862091, 2009.

Demers, L.-A. A. and Gosselin, C.: Kinematic design of a thumb metacarpal for an anthropomorphic robotic hand, in: Proceedings of the ASME International Design Engineering Technical Conferences (IDETC 2010), Montréal, Canada, 15-18 August 2010.

Gurbuz, H., Mesut, R., and Nesrin Turan, F.: Measurement of active abduction of metacarpophalangeal joints via electronic digital incinometric technique, Ital. J. Anat. Embryol., 111, 9-14, 2006.

Kapandji, A. I.: Physiologie articulaire : schémas commentés de mécanique humaine, Vol. 1, Maloine, 6 Edn., 2005.

Norkin, C. C. and White, D. J.: Measurement of Joint Motion: A Guide to Goniometry, Philadelphia, 4 Edn., 2009.

Smahel, Z. and Klímová, A.: The influence of age and exercise on the mobility of hand joints: 1: Metacarpophalangeal joints of the three-phalangeal fingers, Acta Chir. Plast., 46, 81-88, 2004.

Tubiana, R. and Thomine, J. M.: La main : Anatomie fonctionnelle et examen clinique, Masson, Paris, 1990. 